\title{
Determining Correlation of Food Intake and Satiety Related Hormones with the Findings of Brain Computerized Tomography (CT) Scans and Psychological Disorders in Children with Mild to Moderate Traumatic Brain Injury
}

Shabnam Bagheri

Shiraz University of Medical Sciences

Mohammad Sadegh Masoudi

Shiraz University of Medical Sciences

Maryam Ekramzadeh ( $\square$ mekramzade@gmail.com )

Shiraz University of Medical Sciences

\section{Research Article}

Keywords: Brain Concussion, Food intake, Appetite, Satiety, Pediatric, Depression, Behavior Disorders, Mental Disorder, Hypocretin, Ghrelin, Leptin, Food craving, Hypothalamus

Posted Date: December 9th, 2020

DOl: https://doi.org/10.21203/rs.3.rs-116514/v1

License: (9) This work is licensed under a Creative Commons Attribution 4.0 International License. Read Full License 


\section{Abstract}

Background: Traumatic brain injury (TBI) is an impairment with high prevalence amongst the pediatric population. Central nervous system (CNS) regulates food intake and each region of the brain can be responsible for appetite control network. It seems that TBI might affect appetite control system which could result in overeating or reduced food intake. Hence, the aim of this study was to determine, if there is a relationship between daily energy intake and satiety related hormones with computerized tomography scan (CT scan) findings in children with mild to moderate TBI.

Methods: In this cross sectional study, 50 pediatrics with mild to moderate TBI were recruited and the following steps were performed for each patient: Completing the demographic information (age, gender, medical disorders, etc.), dietary intake assessment, taking blood samples for biochemical evaluations, and evaluating psychological disorders using the "Rutter Children Behavior Questionnaire". The patient's CT scan was also used to determine the main area of brain injury.

Results: The results showed that in comparison with the Reference Dietary Allowance (RDA) values, calorie intake was inadequate in $32 \%$ of the patients, while $32 \%$ had excess calorie intake. Comparison of the mean daily energy intake and serum levels of satiety hormones in different categorical CT scan findings showed that there was a significant relationship between energy intake and the site of injury. Post- hoc analysis showed that energy intake in patients with temporal lobe injury was significantly higher than in patients with frontal lobe injury or injuries to other sites of the brain. The results also showed that mean serum Ghrelin and Orexin levels in patients with temporal lobe injury were higher than patients with frontal lobe injury but no significant relationship was found regarding leptin and site of injury. Moreover, it was shown that children with frontal lobe injury had significantly more behavioral disorders.

Conclusions: It seems that frontal lobe injuries might be associated with anorexia while temporal lobe injury can be associated with increased food intake. Future studies are recommended to determine, if there is a relationship between satiety related hormones with other site of injury in TBI patients.

\section{Background}

Traumatic brain injury (TBI) is a temporary or permanent impairment of the brain function, caused by direct or indirect hit to the head. TBI refers to one or a combination of brain injuries, such as skull fracture, extra-cranial and intra-parenchymal hemorrhage (1). Brain injuries are divided into three groups of Concussion, Contusion and Diffused axonal injury (2). TBI is classified into three groups of mild, moderate and severe according to the Glasgow coma Scale (GCS). Accordingly, GCS 13-15 is considered as mild, GCS 9-12 as moderate and GCS 3-8 as severe injuries (3).

Recent data from the Centers for Disease Control and Prevention (CDC) showed that the TBI statistics have increased by $58 \%$ over the past 10 years (4), and it is the third leading cause of death and disability in the world in 2020 (5). The average rate of mortality for patients with severe TBI is $39 \%$, and those who 
survive have significant disabilities, which itself is another socioeconomic concern (6). In children and adolescents, TBI is the first cause of death and disability in the United States (7). Annually, about 500,000 children under the age of 14 suffer from head injuries (8). As far as we know, there is no statistics on the brain trauma and its costs in Iran. But it seems that Iran is amongst the top countries in the world to have trauma accidents, most of which lead to brain injuries (9).

Patients with moderate to severe brain injury are often unable to meet their nutritional needs. Skull fracture might lead to a damage in skull-based nerves, which are associated with chewing, swallowing, smelling and tasting. In addition, fractures of the face and teeth can delay the onset of oral nutrition (2). For such patients, inadequate intake of nutrients is due to anorexia, early satiety, problems with taste and olfactory senses, inactivity, depression and dysphagia or complications such as aspiration (10).

The central nervous system (CNS) regulates food intake and the sense of hunger or satiety, and each region is a part of a complex network in appetite control; hence, it can be expected that in brain injury, a disruption will happen in appetite and food intake control. The ventromedial hypothalamus is the center of satiety while the lateral hypothalamus is the center for desire to eat; thus, the damage to the ventromedial hypothalamus can increase the desire to eat and vice versa the lateral hypothalamic injury can reduce the tendency to eat (11). Findings also showed that the damage to the ventricular nuclei, thalamic vessels and hippocampus can lead to overeating (12). On the other hand, brain stem injury might decrease appetite (13-15). Numerous studies also suggested that the damage to the frontal lobe reduces the desire to eat (16). since the amygdala and hippocampus have role in food intake regulation and appetite control, temporal lobe damage might lead to increased food intake (17). Findings suggest that following $\mathrm{TBI}$, the changes in eating behavior might result in overeating or anorexia (18).

Therefore, the aim of this study for the first time was to determine whether there is a relationship between daily energy intake, satiety related hormones, and the degree and site of brain damage in children with mild to moderate TBI, using CT scan imaging.

\section{Methods}

\section{Data collection and sample size:}

In this cross sectional study, pediatrics in the age range of 5-15 years old with mild to moderate TBI referred to the pediatric neurosurgery clinic affiliated with Shiraz University of Medical Sciences were enrolled. The sample size was calculated to be 50 patients based on the energy intake value in pelizzo et al. study with the power of $80 \%$ and $a=5 \%: \quad n 1=n 2=\frac{2 \sigma^{2}\left(z_{2}^{\alpha}+\mathbf{z} \boldsymbol{\beta}\right)^{2}}{(\boldsymbol{\mu 1} \mathbf{\mu} \mathbf{2})^{2}}$ (19). This study was approved by ethics committee of Shiraz University of Medical Sciences. (No. IR.SUMS.REC.1397.708) and followed the principles of the Declaration of Helsinki.

\section{Inclusion criteria:}


children of 5-15 years old with mild to moderate TBI who were injured within the past 3-6 months without any other illnesses that could affect their food intake, such as cancer, liver and kidney failure, thyroid disorders, and diabetes were recruited.

\section{Exclusion criteria:}

Patients who were on parenteral nutrition, suffered multiple injuries or had a history of neurological or psychiatric problems, used any medications or supplements that could affect appetite and also were reluctant to participate in the study, were excluded.

After explaining the research objectives to the childrens' parents, they were asked to complete the consent form and the following steps were performed for each patient: Completing the demographic information (age, gender, medications, medical disorders), dietary intake assessment, taking blood samples for biochemical evaluations, assessing psychological disorders using the "Rutter Children Behavior Questionnaire" (the parent's form) and doing CT scan for determining the main area of brain injury (frontal, temporal, occipital, or other sites of brain).

\section{Dietary intake assessment:}

to assess the children's dietary intake, data from three $24 \mathrm{~h}$ dietary recalls were taken. Two assessments were made on weekdays and a third one during the weekends. The data were assessed under the guidance of a skilled dietitian. The reported values were then entered into the Nutritionist 4 software. The average energy intake was then compared with the RDA values for each individual (4). Adequate energy intake was defined as intake 75 to $110 \%$ of the RDA, while inadequate and excess intake were considered as intakes less than $75 \%$ and more than $110 \%$ of the RDA, respectively (20).

\section{Evaluation of behavioral disorders:}

Children were assessed for behavioral disorders using the "Rutter Children Behavior Questionnaire" (the parent form), which was provided to the child's parents to answer the questions. It should be noted that the Rutter Behavioral Problems Questionnaire was developed by Michael Rutter in 1975, which is consisted of 31 items including 13 questions related to general health, habits and lifestyle (including complaints of headache, stomach pain with nausea, shortness of breath, urinary or fecal incontinency, refusal to go to school, speech problems, stealing behaviors, and eating and sleeping problems) and 18 questions related to child behavioral disorders (including behaviors such as restlessness, aggression, irritability, depression, disobedience, concentration problems, and lying) and is completed by parents. A score of 13 is the cut-off point, and children with a score of 13 or higher are considered as having behavioral disorders. (21). 


\section{CT scan evaluation:}

evaluation and interpretation of the patients' CT scan was done by the neurosurgeon to determine where the affected region of the brain was (frontal, temporal, parietal, occipital lobe, or other sites of brain) and if the cerebral hemorrhage existed. By referring to the patient's medical history, information on the day that the trauma had occurred, the length of hospitalization, the cause of trauma, the GCS at admission and also the therapeutic strategies were recorded.

\section{Biochemical assessment:}

Fasting blood samples were taken from each participant. The serums were prepared by centrifuging at $3000 \mathrm{rpm}$ for 15 minutes and were stored at $-70^{\circ} \mathrm{C}$ until further analysis. Serum leptin was measured by ELISA method, using German Mediagnost kit, serum Ghrelin was measured by ELISA method, using Chinese Crystallday kit, and serum Orexin A was measured by ELISA method, using Chinese Crystallday kit.

\section{Statistical Analysis:}

Data analysis was done, using SPSS software (version 24). Quantitative variables were reported as mean \pm SD. The normality of quantitative variables was evaluated by Kolmogorov-Smirnov test. To compare the mean \pm SD of normal quantitative variables by multivariate qualitative variables, one-way ANOVA was used and Kruskal-Wallis test, if the variables were not normal. Additional post-hoc tests were also used to compare the groups two by two. Comparison of variables in two groups according to Rutter test score were done by independent sample t-test (for normal data) and Mann Whitney test for nonparametric ones. $\mathrm{P}<0.05$ was considered to be statistically significant.

\section{Results}

In this study of fifty patients (36 boys and 14 girls) with mild to moderate TBI were examined. The mean age (years), GCS at admission and length of hospitalization (day) of the patients was $8.82 \pm 3.12,10.12$ \pm 1.91 and $12.03 \pm 17.26$, respectively. (Table 1 ) 
Table 1

Demographic characteristics and biochemical variables in pediatrics with TBI.

\begin{tabular}{|lllll|}
\hline Variable & Total (50) & Boys (36) & Girls (14) & p-value \\
\hline Age (years) & $8.82 \pm 3.12$ & $8.91 \pm 0.51$ & $8.43 \pm 0.88$ & 0.65 \\
\hline GCS at admission & $10.12 \pm 1.91$ & $9.97 \pm 1.89$ & $10.50 \pm 1.99$ & 0.40 \\
\hline $\begin{array}{l}\text { length of hospitalization } \\
\text { (days) }\end{array}$ & $12.03 \pm 17.26$ & $11.55 \pm 15.55$ & $13.26 \pm 21.67$ & 0.42 \\
\hline Leptin $(\mathrm{ng} / \mathrm{ml})$ & $4.82 \pm 6.51$ & $4.80 \pm 6.78$ & $4.87 \pm 6.00$ & 0.45 \\
\hline Ghrelin $(\mathrm{ng} / \mathrm{ml})$ & $2.95 \pm 3.71$ & $2.89 \pm 3.74$ & $3.12 \pm 3.74$ & 0.97 \\
\hline Orexin A $(\mathrm{pg} / \mathrm{ml})$ & $593.00 \pm 552.64$ & $576.00 \pm$ & $636.74 \pm$ & 0.76 \\
\hline $\begin{array}{l}\text { Energy intake }(\mathrm{kcal} / \text { day) } \\
\text { Energy intake adequacy }(\%) \text { ** }\end{array}$ & $\mathbf{1 5 1 4 . 5 6 \pm}$ & $1622.4 \pm 640.7$ & $1237.3 \pm 302.2$ & 0.4 \\
\hline Rutter test score & 589.86 & & & 0.6 \\
\hline * The data are reported as mean \pm SD. & $17.2 \pm 8.3$ & $17.2 \pm 7.9$ & $17.3 \pm 9.6$ & 0.5 \\
\hline ** $<0.05$ was considered as significant. & & & \\
\hline
\end{tabular}

Based on the GCS, most of the patients (88\%) suffered from moderate TBI. The majority of them were taking anticonvulsant medications (levebel or phenytoin). Sleep disorder and headache were reported among the most prevalent medical disorders in this population. Cerebral hemorrhage was observed in $28 \%$ of the children, most of which was related to the epidural hematoma. Most of the participants (64\%) did not need surgery after TBI. About sixty-eight percent of the children had Rutter test score more than 13 , which indicates the existence of psychological disorders. In this study, the most common cause of trauma was car accident and the most prevalent affected injury site was frontal and temporal lobe of the brain. (Table 2) 
Table 2

Medical records and CT scan findings of the patients.

\begin{tabular}{|c|c|c|c|}
\hline Variable & Total (50) & Boys (36) & Girls (14) \\
\hline \multicolumn{4}{|l|}{ Severity of injury } \\
\hline Mild & $6(12)$ & $4(11.1)$ & $2(14.3)$ \\
\hline Moderate & $44(88)$ & $32(88.9)$ & $12(85.7)$ \\
\hline \multicolumn{4}{|l|}{ Medical disorders } \\
\hline No medical disorders & $8(16)$ & $6(16.7)$ & $2(14.3)$ \\
\hline Seizure & $6(12)$ & $4(11.1)$ & $2(14.3)$ \\
\hline Headache & $18(36)$ & $13(36.1)$ & $5(35.7)$ \\
\hline Sleep disorders & $18(36)$ & $13(36.1)$ & $5(35.7)$ \\
\hline \multicolumn{4}{|l|}{ Medicine } \\
\hline Levebel & $38(76)$ & $28(77.8)$ & $10(71.4)$ \\
\hline Phenytoin & $6(12)$ & $4(11.1)$ & $2(14.3)$ \\
\hline No medication & $6(12)$ & $4(11.1)$ & $2(14.3)$ \\
\hline \multicolumn{4}{|l|}{ Cerebral hemorrhage } \\
\hline No hemorrhage & $36(72)$ & $24(66.7)$ & $12(85.7)$ \\
\hline Epidural & $12(24)$ & $10(27.8)$ & $2(14.3)$ \\
\hline Subdural & $1(2)$ & $1(2.8)$ & $0(0)$ \\
\hline Intraventricular & $1(2)$ & $1(2.8)$ & $0(0)$ \\
\hline \multicolumn{4}{|l|}{ Cause of trauma } \\
\hline Car accident & $32(64)$ & $22(61.1)$ & $10(71.4)$ \\
\hline Motor accident & $8(16)$ & $6(16.7)$ & $2(14.3)$ \\
\hline Falling & $7(14)$ & $5(13.9)$ & $2(14.3)$ \\
\hline Penetration of an objective & $3(6)$ & $3(8.3)$ & $0(0)$ \\
\hline \multicolumn{4}{|l|}{ Surgery } \\
\hline Yes & $18(36)$ & $14(38.9)$ & $4(28.6)$ \\
\hline No & $32(64)$ & $22(61.1)$ & $10(71.4)$ \\
\hline \multicolumn{4}{|l|}{ Rutters test score } \\
\hline Less than 13 & $16(32)$ & $10(27.8)$ & $6(42.9)$ \\
\hline
\end{tabular}




\begin{tabular}{|lccc|}
\hline Site of the injury & $34(68)$ & $26(72.2)$ & $8(57.1)$ \\
\hline Frontal lobe & $18(36)$ & $13(36.1)$ & $5(35.7)$ \\
\hline Temporal lobe & $17(34)$ & $13(36.1)$ & $4(28.6)$ \\
\hline Skull base fracture & $7(14)$ & $4(11.1)$ & $3(21.4)$ \\
\hline other areas of the brain & $8(16)$ & $6(16.7)$ & $2(14.3)$ \\
\hline *Data are reported as frequency (percent). & & \\
\hline
\end{tabular}

The results showed that in comparison with the RDA values (2), calorie intake was inadequate in $32 \%$ of the patients, while $32 \%$ had excess energy intake. (Table 3 )

Table 3

Energy intake of the patients in comparison with the reference values

\begin{tabular}{|llll|}
\hline Calorie intake & Total (50) & Boys (36) & Girls (14) \\
\hline$<75 \%$ of RDA & $16(32)$ & $12(33.3)$ & $4(28.6)$ \\
\hline $75-110 \%$ of RDA & $18(36)$ & $11(30.5)$ & $7(50)$ \\
\hline$>110 \%$ of RDA & $16(32)$ & $13(36.1)$ & $3(21.4)$ \\
\hline *Data are reported as frequency (percent). \\
\hline
\end{tabular}

Based on the comparison of mean daily energy intake and serum levels of related satiety hormones in different categorical CT scan findings, there was a significant relationship between energy intake and the site of injury ( $p$ <.001). Post- hoc analysis showed that energy intake in patients with temporal lobe injury was higher than patients with frontal lobe injury, skull base fracture or injuries to other sites of brain, meaning that overeating is more likely to happen in parietal lobe injuries. There was also a significant relationship between serum Ghrelin and Orexin A levels with the site of injury. According to post- hoc analysis mean serum Ghrelin $(p=0.02)$ and Orexin $A(p=0.04)$ levels in patients with temporal lobe injury were higher than patients with frontal lobe injury. No statistically significant relationship was found regarding leptin and site of injury. Furthermore, a significant relationship was found between Rutter test score and the area of brain injury $(p=0.03)$. Children with frontal lobe injury had a higher Rutter test score, indicating that behavioral disorders are more common in those with frontal damage. (Table 4) 
Table 4

comparison of the mean energy intake, satiety hormones and Rutter test score with CT scan findings

\begin{tabular}{|c|c|c|c|c|c|}
\hline Variable & $\begin{array}{l}\text { Frontal } \\
\text { injury }\end{array}$ & $\begin{array}{l}\text { Temporal } \\
\text { injury }\end{array}$ & $\begin{array}{l}\text { Skull base } \\
\text { fracture }\end{array}$ & $\begin{array}{l}\text { Injuries of the } \\
\text { other sites }\end{array}$ & $\begin{array}{l}\text { p- } \\
\text { value* }\end{array}$ \\
\hline Energy intake & $\begin{array}{l}1234.07 \pm \\
605.93\end{array}$ & $\begin{array}{l}1938.27 \\
\pm 481.49\end{array}$ & $\begin{array}{l}1312.54 \\
\pm 280.74\end{array}$ & $1422.02 \pm 507.53$ & $\begin{array}{l}< \\
0.001\end{array}$ \\
\hline $\begin{array}{l}\text { Energy intake } \\
\text { adequacy } \star \star\end{array}$ & $\begin{array}{l}67.01 \\
\pm 17.49\end{array}$ & $\begin{array}{l}122.50 \\
\pm 17.98\end{array}$ & $79.55 \pm 8.62$ & $74.83 \pm 7.87$ & $\hat{0}_{0.001}$ \\
\hline Leptin & $6.21 \pm 7.80$ & $3.35 \pm 5.62$ & $2.83 \pm 1.36$ & $6.5 \pm 7.60$ & 0.19 \\
\hline Ghrelin & $1.84 \pm 2.96$ & $5.43 \pm 4.67$ & $1.88 \pm 1.37$ & $1.13 \pm 0.49$ & 0.006 \\
\hline Orexin A & $\begin{array}{l}374.04 \\
\pm 239.80\end{array}$ & $\begin{array}{l}1000.87 \\
\pm 751.11\end{array}$ & $\begin{array}{l}472.81 \\
\pm 280.68\end{array}$ & $324.11 \pm 129.68$ & 0.01 \\
\hline Rutter test score & $20.83 \pm 7.47$ & $13.47 \pm 7.88$ & $19.85 \pm 9.8$ & $14.62 \pm 6.43$ & 0.03 \\
\hline
\end{tabular}

Comparing energy intake and serum levels of satiety hormones in two groups of TBI children according to Rutter test score, showed that those with a higher Rutter score (which is indicative of more severe behavioral problems), had significantly lower levels of Orexin, Ghrelin and energy intake than those with lower scores. (Table 5)

Table 5

Comparison of energy intake and serum levels of satiety hormones with Rutter test score

\begin{tabular}{|llll|}
\hline Variable & $\begin{array}{l}\text { Rutter score }<13 \\
(\mathbf{n = 1 6})\end{array}$ & $\begin{array}{l}\text { Rutter score }>13 \\
(\mathbf{n}=34)\end{array}$ & p-value* \\
\hline Energy intake $(\mathrm{kcal} /$ day $)$ & $1709.63 \pm 691.6$ & $1422.75 \pm 521.63$ & 0.1 \\
\hline Energy intake adequacy $(\%) * \star$ & $107.71 \pm 29.28$ & $80.02 \pm 24.66$ & 0.002 \\
\hline Leptin $(\mathrm{ng} / \mathrm{ml})$ & $2.46 \pm 3.15$ & $5.92 \pm 7.38$ & 0.15 \\
\hline Ghrelin $(\mathrm{ng} / \mathrm{ml})$ & $4.9 \pm 4.43$ & $2.04 \pm 2.96$ & 0.03 \\
\hline Orexin $\mathrm{A}(\mathrm{pg} / \mathrm{ml})$ & $985 \pm 666.69$ & $408.53 \pm 376.69$ & 0.001 \\
\hline *Data are reported as mean \pm SD. & & \\
\hline **Ratio of individuals' energy intake compared to the RDA value. & \\
\hline
\end{tabular}




\section{Discussion}

\section{Correlation of food intake and satiety related hormones with CT scan findings}

The current study showed that in comparison with RDA values, calorie intake was inadequate in $32 \%$ of the patients while $32 \%$ had excess energy intake. In addition, there was a significant relationship between energy intake and the site of injury. There was also a significant relationship between serum Ghrelin and Orexin levels with the site of injury. To the best of our knowledge, this is the first study to examine the relationship between satiety/ hunger hormone levels with the site of injury in children with traumatic brain injury. In a study by Pelizzo et al. on pediatrics with neurological disorders, the results showed that daily energy intake was lower than the expected needed level in $23.8 \%$ and higher than normal in $50 \%$ of the patients (22).

Our findings also indicated that there was a significant relationship between energy intake and the affected area of brain based on CT scan findings. Energy intake in patients with temporal lobe injury, was significantly higher than in patients with injuries to other sites of brain and it was also shown that energy intake in patients with frontal lobe injury was significantly lower than in patients with injuries to other sites of brain.

In line with the results of this study, Goddard et al. in a case report of a 25 year-old man, showed the association between glioma injury of the right frontal lobe and hypophagia or anorexia nervosa (16). While in a case study on a 42-year-old man, Das et al. showed a correlation between frontal lobe injury caused by a road accident with severe overeating behavior, and reported that the patient's weight increased from $78 \mathrm{~kg}$ to $108 \mathrm{~kg}$ within a year (23). A case report by Levine et al. on two patients also showed over eating behaviors in a patient with occipital and right temporal injury and hypophagia behaviors in a patient with encephalomalacia in temporal and right frontal (24). Furthermore, a case report by Castano et al. reviewed eating disorders in four patients with severe TBI, indicated hypophagia behaviors in patients with frontal lobe, thalamus and diffused axonal injuries, and overeating behaviors in patients with temporal and hypothalamus injuries (25).

As mentioned earlier, the CNS regulates food intake and each region of the brain is responsible for eating behavior and appetite control. Therefore, it can be expected that brain injury can disrupt the regulation of food intake. It seems that frontal lobe injury might be associated with anorexia and hypophagia behaviors, and temporal lobe injury with over eating. The temporal lobe of the brain consists of two important parts of amygdala and hippocampus that play important roles in food intake and body weight regulation. Therefore, regulation of satiety could be impaired following temporal lobe injury, which may be an explanation for excessive food intake (17). On the other hand, the frontal lobe has motor and premotor areas, as well as the Broca area, which controls the behavior, decision making and emotional outbursts (26). As it was also shown in the current study, children with frontal lobe injury had a higher Rutter test score, indicating more behavioral problems in patients with damage to this site of brain. 
Therefore, one of the causes of anorexia in patients with frontal lobe injury might be associated with cognitive problems and depression that may lead to decreased food intake secondary to psychological impairment, especially post-traumatic depression (27).

Moreover, in the current study, the mean serum levels of Ghrelin and Orexin were significantly higher in children with temporal lobe injury than in children with frontal lobe injury, which might be another reason for higher calorie intake and better appetite in patients with temporal lobe injury. As we know Ghrelin is called a hunger hormone increasing food intake released mainly from gastrointestinal tract. It also plays a role in improving learning and memory by regulating synaptic terminals and producing synapses in the hypothalamus and hippocampus (28). Interestingly, the ghrelin receptor is found in the basolateral complex of the amygdala, a brain region that is located in temporal lobe and play important role in regulating food intake (29). Orexin (hypocretin) is also a neuropeptide that regulates appetite and feeding behavior. It stimulates food intake and increase the craving for food. Orexin also activates orexin neurons, monoaminergic and cholinergic neurons in the hypothalamus/brainstem regions. The activities of monoaminergic neurons are known to be associated with sleep and awakeness. Decreased levels of orexin has been shown to be related to behavioral problems as well as sleep disorders (30). Orexin is also produced by the lateral hypothalamus and the orexin receptor (OX1R) is expressed in many regions of the brain, including the prefrontal cortex (31), which may be an explanation for loss of orexin production from neurons following TBI.

\section{Correlation of food intake and satiety related hormones with psychological disorders}

Our findings also demonstrated that children with frontal lobe injury had more behavioral problems and these children had significantly lower levels of Orexin and Ghrelin too.Thus children with more severe behavioral problems, had significantly lower food intake. As mentioned earlier, the frontal lobe is consisted of motor and pre-motor areas and the Broca area, which controls behavior that may be an explanation for psychological disorders following frontal lobe injury (26). Therefore, one of the causes of decreased food intake in patients with frontal lobe injury might be due to cognitive problems and depression. (27).

In a study by Fedoroff JP et al a significant relationship between lesions in prefrontal cortex and depression was found in the acute phase of TBI (32). In another study in patients with TBI, the results indicated that plasma ghrelin levels were significantly lower in patients with cognitive disorders than in patients without cognitive disorders and plasma ghrelin level was an independent predictor of cognitive disorders 3 months after TBI (33).

\section{Limitations}


Small sample size and the absence of control group are the limitations of the current study. Difficulty in taking blood samples for these groups of patients was another drawback in our research. More studies are warranted to determine the correlation of serum growth hormone, insulin like growth factor 1 (IGF-1), thyroid stimulating hormone (TSH), and weight changes with the findings of CT scans in children with traumatic brain injury. Also more detailed studies are needed to assess nutritional consequences of TBI patients with regard to different sites of brain injury.

\section{Conclusions}

Traumatic brain injury might disrupt food intake. It seems that frontal lobe injury is associated with anorexia and hypophagia behaviors and temporal lobe injury might be associated with increased food intake. More studies are required to determine, if there is a relationship between satiety related hormones and the exact area of brain injury in these patients.

\section{List Of Abbreviations}

TBI (Traumatic Brain Injury), CNS (Central nervous system), CT scan (Computerized tomography scan), RDA (Reference Dietary Allowance), GCS (Glasgow coma scale), CDC (Centers for Disease Control and Prevention)

\section{Declarations}

\section{Ethics approval and consent to participate:}

This human study has been approved by research ethics committee of Shiraz University of Medical Sciences. Committee's Reference Number: (No. IR.SUMS.REC.1397.708). All the parents of children signed a written informed consent form after explaining the aim, method and goal of the study for participation.

\section{Consent for publication:}

Not applicable.

\section{Availability of data and materials:}

The datasets used and/or analyzed during the current study are available from the corresponding author on reasonable request.

\section{Competing interests:}


The authors declare that they have no competing interests

\section{Funding:}

This study was funded by grant from Shiraz University of Medical Sciences, Shiraz, Iran (Grant number: 1396-01-84-16191).

\section{Authors' contributions:}

All authors have read and approved the manuscript. SHB wrote original draft, Data curation; Formal analysis, Investigation, Software, Visualization. MSM: Conceptualization; Methodology; Supervision; Validation; Visualization; review \& editing draft. ME: Conceptualization; Data curation; Formal analysis; Funding acquisition; Methodology; Project administration; Supervision; Validation; Visualization; Writing original draft; review \& editing draft.

Correspondence to: Maryam Ekramzadeh (mekramzade@gmail.com)

\section{Acknowledgement:}

The authors would like to thank the Vice Chancellery of Research, Shiraz University of Medical Sciences, for its financial support. The authors also wish to thank the Research Consultation Center (RCC) at Shiraz University of Medical Sciences for their assistance in editing this manuscript.

\section{References}

1. Scorza KA, Raleigh MF, O'Connor FG. Current concepts in concussion: evaluation and management. American family physician. 2012;85(2):123-32.

2. MAHAN LK RJ. KRAUS E'S FOOD \& THE NUTRITION CARE PROCESS. 2017(Elsevier):824-6

3. Stern TAF MW, Timothy E; Rosenbaum, Jerrold F. Massachusetts General Hospital Comprehensive 2016;82(Clinical Psychiatry):883-95.

4. Baltazar GA, Pate AJ, Panigrahi B, LaBoy S, Prosniak R, Mody A, et al. Malnutrition as measured by albumin and prealbumin on admission is associated with poor outcomes after severe traumatic brain injury. The American surgeon. 2015;81(2):E61-3.

5. Sharma B, Lawrence DW, Hutchison MG. Branched Chain Amino Acids (BCAAs) and Traumatic Brain Injury: A Systematic Review. The Journal of head trauma rehabilitation. 2018;33(1):33-45.

6. Wang D, Zheng SQ, Chen XC, Jiang SW, Chen HB. Comparisons between small intestinal and gastric feeding in severe traumatic brain injury: a systematic review and meta-analysis of randomized controlled trials. Journal of neurosurgery. 2015;123(5):1194-201. 
7. Pu H, Jiang X, Wei Z, Hong D, Hassan S, Zhang W, et al. Repetitive and Prolonged Omega-3 Fatty Acid Treatment After Traumatic Brain Injury Enhances Long-Term Tissue Restoration and Cognitive Recovery. Cell transplantation. 2017;26(4):555-69.

8. Guilliams K, Wainwright MS. Pathophysiology and Management of Moderate and Severe Traumatic Brain Injury in Children. Journal of child neurology. 2016;31(1):35-45.

9. Zink BJ, Szmydynger-Chodobska J, Chodobski A. Emerging concepts in the pathophysiology of traumatic brain injury. The Psychiatric clinics of North America. 2010;33(4):741-56.

10. Dionyssiotis Y, Papachristos A, Petropoulou K, Papathanasiou J, Papagelopoulos P. Nutritional Alterations Associated with Neurological and Neurosurgical Diseases. The open neurology journal. 2016;10:32-41.

11. Perry B, Wang Y. Appetite regulation and weight control: the role of gut hormones. Nutrition \& diabetes. 2012;2:e26.

12. JEH ACG. Textbook of medical physiology. 2006(ELSEVIER SAUNDERS):867-72.

13. Rm U, Treasure J. Brain lesions and eating disorders. Journal of neurology, neurosurgery, and psychiatry. 2005;76:852-7.

14. Farr OM, Li CR, Mantzoros CS. Central nervous system regulation of eating: Insights from human brain imaging. Metabolism: clinical and experimental. 2016;65(5):699-713.

15. Heisler LK, Lam DD. An appetite for life: brain regulation of hunger and satiety. Current opinion in pharmacology. 2017;37:100-6.

16. Goddard E, Ashkan K, Farrimond S, Bunnage M, Treasure J. Right frontal lobe glioma presenting as anorexia nervosa: further evidence implicating dorsal anterior cingulate as an area of dysfunction. The International journal of eating disorders. 2013;46(2):189-92.

17. Coppin G. The anterior medial temporal lobes: Their role in food intake and body weight regulation. Physiology \& behavior. 2016;167:60-70.

18. Crenn P, Hamchaoui S, Bourget-Massari A, Hanachi M, Melchior JC, Azouvi P. Changes in weight after traumatic brain injury in adult patients: a longitudinal study. Clinical nutrition (Edinburgh, Scotland). 2014;33(2):348-53.

19. Pelizzo G, Calcaterra V, Carlini V, Fusillo M, Manuelli M, Klersy C, et al. Nutritional status and metabolic profile in neurologically impaired pediatric surgical patients. Journal of pediatric endocrinology \& metabolism : JPEM. 2017;30.

20. Gupta A, Noronha J, Kamath S, Garg M. Dietary intake of macronutrients and micronutrients among adolescent girls: A cross sectional study. Clinical Epidemiology and Global Health. 2018;6.

21. Elander J, Rutter M. Use and development of the Rutter parents' and teachers' scales. International Journal of Methods in Psychiatric Research. 1996;6(2):63-78.

22. Pelizzo G, Calcaterra V, Carlini V, Fusillo M, Manuelli M, Klersy C, et al. Nutritional status and metabolic profile in neurologically impaired pediatric surgical patients. Journal of pediatric endocrinology \& metabolism : JPEM. 2017;30(3):289-300. 
23. Das A, Elwadhi D, Gupta M. Secondary Eating Disorder: A Reality? Case Report of Post Brain Injury Sequelae. Indian journal of psychological medicine. 2017;39(2):205-8.

24. Levine R, Lipson S, Devinsky O. Resolution of eating disorders after right temporal lesions. Epilepsy \& behavior : E\&B. 2003;4(6):781-3.

25. Castano B, Capdevila E. Eating disorders in patients with traumatic brain injury: a report of four cases. NeuroRehabilitation. 2010;27(2):113-6.

26. DiGiulio M. JD KJ. Medical-Surgical Nursing: Demystified. 2016(McGraw-Hill).

27. Gouick J, Gentleman D. The emotional and behavioural consequences of traumatic brain injury. Trauma. 2004;6(4):285-92.

28. Gómez-Pinilla F. Brain foods: The effects of nutrients on brain function. Nature reviews Neuroscience. 2008;9:568-78.

29. Meyer RM, Burgos-Robles A, Liu E, Correia SS, Goosens KA. A ghrelin-growth hormone axis drives stress-induced vulnerability to enhanced fear. Molecular Psychiatry. 2014;19(12):1284-94.

30. Inutsuka A, Yamanaka A. The physiological role of orexin/hypocretin neurons in the regulation of sleep/wakefulness and neuroendocrine functions. Frontiers in endocrinology. 2013;4:18.

31. Dong $X, H u Y$, Feng $Z$. Wake-promoting effects of vagus nerve stimulation after traumatic brain injury: Upregulation of orexin-A and orexin receptor type 1 expression in the prefrontal cortex. Neural Regeneration Research. 2018;13.

32. Fedoroff JP, Starkstein SE, Forrester AW, Geisler FH, Jorge RE, Arndt SV, et al. Depression in patients with acute traumatic brain injury. The American journal of psychiatry. 1992;149(7):918-23.

33. Xu Z, Lv X-A, Wang J-W, Chen Z-P, Qiu H-S. Predictive value of early decreased plasma ghrelin level for three-month cognitive deterioration in patients with mild traumatic brain injury. Peptides. 2014;54:180-5. 